\title{
Evaluation of Approaches to Deal with Low-Frequency Nuisance Covariates in Population Pharmacokinetic Analyses
}

\author{
Chakradhar V. Lagishetty ${ }^{1,2}$ and Stephen B. Duffull ${ }^{1}$
}

Received 21 January 2015; accepted 27 May 2015; published online 26 June 2015

\begin{abstract}
Clinical studies include occurrences of rare variables, like genotypes, which due to their frequency and strength render their effects difficult to estimate from a dataset. Variables that influence the estimated value of a model-based parameter are termed covariates. It is often difficult to determine if such an effect is significant, since type I error can be inflated when the covariate is rare. Their presence may have either an insubstantial effect on the parameters of interest, hence are ignorable, or conversely they may be influential and therefore non-ignorable. In the case that these covariate effects cannot be estimated due to power and are non-ignorable, then these are considered nuisance, in that they have to be considered but due to type 1 error are of limited interest. This study assesses methods of handling nuisance covariate effects. The specific objectives include (1) calibrating the frequency of a covariate that is associated with type 1 error inflation, (2) calibrating its strength that renders it non-ignorable and (3) evaluating methods for handling these non-ignorable covariates in a nonlinear mixed effects model setting. Type 1 error was determined for the Wald test. Methods considered for handling the nuisance covariate effects were case deletion, Box-Cox transformation and inclusion of a specific fixed effects parameter. Non-ignorable nuisance covariates were found to be effectively handled through addition of a fixed effect parameter.
\end{abstract}

KEY WORDS: Box-Cox transformation; case deletion and pharmacometrics; fixed effect parameter; nuisance covariate.

\section{INTRODUCTION}

The aim of pharmacokinetic (PK) analyses is to understand the time course of drug concentration exposure in order to predict drug response. The population approach, using nonlinear mixed effect modelling, is commonly used for PK analyses and provides an estimate of the parameters of the underlying structural PK model and the variance of betweensubject effects. In these analyses, it is also one of the aims to identify potential covariates (patient characteristics that influence the value of an individual's parameter estimates). These analyses are often implemented in data that arise from late phase 3 clinical studies or post-marketing studies which may be collected across multiple centres. The data collected from such clinical studies may include rare factors such as unexpected concomitant interacting medicines and/or genotypes in the form of binary covariates. The rare occurrence of these covariates creates a dilemma as to how best to incorporate the information from the covariate. In the first instance, as the frequency of the covariate approaches zero,

\footnotetext{
${ }^{1}$ School of Pharmacy, University of Otago, Dunedin, New Zealand.

${ }^{2}$ To whom correspondence should be addressed. (e-mail: chakradharshetty@gmail.com)
}

then the false positive rate increases (type 1 error inflation) (1). The inflated type 1 error rate creates a covariate whose influence cannot be differentiated from the null. Others have explored the type 1 error rate of low-frequency covariates and shown inflation of type 1 error rate in a population analysis framework (1-5). These works have also shown that the choice of estimation algorithm in NONMEM will also influence the type 1 error inflation. Alternative approaches are proposed for the increase of type 1 error for asymptotic test for small sample size. The permutation test could be used as a correction measure in such circumstances $(2,6)$.

One method of dealing with low-frequency covariates is to ignore them in the analysis by including the patient but not including the covariate. However, this is reasonable only if the covariate is not influential in the analysis. However, if the effect of the covariate is strongly influential, then ignoring the covariate will bias the analysis (either at the level of the fixed effects or variance of the random effects parameter values). Under these circumstances, the covariate is poorly estimable statistically and non-ignorable. We term this covariate nuisance since we cannot account for its influence accurately and cannot ignore it in the analysis. The work of Dansirikul et al. (7) considers a case where incorporation of the covariate resulted in significant inflation of the between-subject variance of clearance. In their work, they used a heavy-tail distribution (multivariate log-t) for the between-subject effects as a pragmatic solution which resulted in a significant 
reduction in the estimate of the variance of the random effects compared to a multivariate lognormal.

The aim of this study was to evaluate approaches to handle nuisance covariates that are non-ignorable in the framework of nonlinear mixed effects modelling. The specific objectives were the following:

1. To calibrate the frequency of the covariate that is associated with type 1 error inflation.

2. To calibrate the strength of the covariate-parameter relationship that renders the covariate non-ignorable and hence nuisance.

3. To assess various methods for handling nuisance covariate effects.

\section{METHODS}

\section{Models and Simulations}

A one compartment PK model with intravenous bolus 'unit' dose was considered as described elsewhere (1). A binary covariate depicting the presence or absence of a particular genotype was considered and randomly simulated with a required probability with sampling replacement. The expected concentration was given as $C_{i j}=f\left(\theta, \eta_{i}, t_{i j}, d_{i}\right)$ where $C_{i j}$ is the expected concentration in the $i$ th subject at the $j$ th time $\left(t_{i j}\right), d_{i}$ denotes dose that we, for simplicity, assume is the same for all individuals, $\theta$ is a $p \times 1$ vector of population parameters and $\eta_{i}$ is a $\mathrm{q} \times 1$ vector of between-subject differences where $p=2$ and $q \leq \mathrm{p}$. The population parameters here are clearance and volume of distribution that relate to the individual parameters via the equation below:

$\boldsymbol{\varphi}_{i}=\boldsymbol{\theta} \times \exp \left(\boldsymbol{\eta}_{\mathbf{i}}\right)$

$\boldsymbol{\eta}_{\mathbf{i}} \stackrel{\mathrm{iid}}{\sim} N(\mathbf{0}, \boldsymbol{\Omega})$ where $\boldsymbol{\Omega}=\left[\begin{array}{cc}\omega_{\mathrm{CL}}^{2} & 0 \\ 0 & \omega_{V}^{2}\end{array}\right]$

Here, $\boldsymbol{\eta}_{\boldsymbol{i}}$ are assumed to be normally distributed centred on zero and variance-covariance matrix of $\Omega(q \times q)$. The diagonal elements of $\Omega$ represent variances of the individual parameter values $\left(\omega^{2}\right)$. Virtual observed concentrations $Y_{i j}$ were then simulated with an additive random error $\left(\varepsilon_{i j}\right)$ such that $Y_{i j}=C_{i j}+\varepsilon_{i j}$ where $\varepsilon_{i j} \stackrel{\text { iid }}{\sim} N\left(0, \sigma^{2}\right)$ and $\sigma^{2}$ is the variance of the residual error. Additive random error was considered for simplicity in the present simulation, and the methods used here should be generalizable to other error model structures. The use of an additive error structure yields the possibility of the simulated observed concentrations being negative. The negative concentrations were accepted in the analysis as if the analytical scientist had reported all concentrations that were extrapolated from the standard curve, even when below the limit of quantification.

Blood sampling design with six observations per each individual at $t=(0,0.001,1.2,3,4$ and 6$)$ hours post-dose was considered (note the units are arbitrary). A total of 100 datasets were simulated for each scenario studied. Each dataset had a pre-determined number of subjects. Note the structural model and design were the same for all simulated scenarios. The parameter values used for simulation were also the initial values for estimation for each specific objective as shown in Table I. The choice of parameter values provides a half-life of $1 \mathrm{~h}$, and therefore, a sampling design to $6 \mathrm{~h}$ provides data over six half-lives.

Simulations were performed using MATLAB software (ver 2012b) and estimation using the software NONMEM ${ }^{\circledR}$ (ver 7.2) using FOCE with INTERACTION.

\section{Calibration of Covariate Frequency that Is Associated with Type 1 Error Inflation}

Population PK data, plasma drug concentrations, were simulated under the null (true) model, i.e. no covariate effect. There were three sample sizes of 20, 100 and 1000 patients with various frequencies of the covariate for each sample size $(50,20,10,5,2$ and $1 \%$ frequencies). For the largest sample size, 1000 subjects, a covariate frequency of $0.1 \%$ was also considered. Each patient provided six plasma drug concentrations. The specific details of parameter values and models are described in Table I under the title of this specific objective. Each simulated dataset was estimated using FOCE with INTERACTION in NONMEM with an alternative model (i.e. using a model with a covariate effect). The decision to reject the null model was based on a Wald test with $p<0.05$. The Wald statistic is calculated using the NONMEM estimates of the covariate coefficient and their asymptotic SE. The Wald statistic follows a chi-square distribution with a $p$ value of 0.05 at a value of 3.84 for 1 degree of freedom. Type 1 error was determined from the proportion of times that the Wald test was rejected.

\section{Calibration of Non-ignorability of a Covariate Effect}

Population PK data were simulated under the full model, i.e. the model that included the influence of the covariate effect. Three scenarios were explored: (1) a weak covariate effect, (2) a moderate covariate effect and (3) a strong covariate effect with the presence of the covariate increasing the value of CL by 20,50 and $100 \%$ respectively. For example, if we consider a typical value of $\mathrm{CL}$ of $1 \mathrm{~L} / \mathrm{h}$, then the CL values for sub-population would be $1.2,1.5$ and $2.0 \mathrm{~L} / \mathrm{h}$ for weak, moderate and strong covariate effect scenarios, respectively. The model parameter values and description of the models used for simulation and estimation are given in Table I with the title of this specific objective. Each of the scenarios of weak, moderate and strong covariate effect considered covariate frequencies of $50,10,5,2$ and $1 \%$. This scenario included a total number of subjects of 100 with 100 replications of each scenario performed. Each of the simulated datasets was estimated using FOCE with INTE RACTION in NONMEM with ignoring the covariate (i.e. using the null model). The mean error (\% relative prediction error) was calculated from the difference of the estimated and nominal values of $\mathrm{CL}$ and variance of the random effect of CL. 
Table I. Parameter Values and Models for Simulation and Estimation of Pharmacokinetic Data for Each Specific Objective in the Study

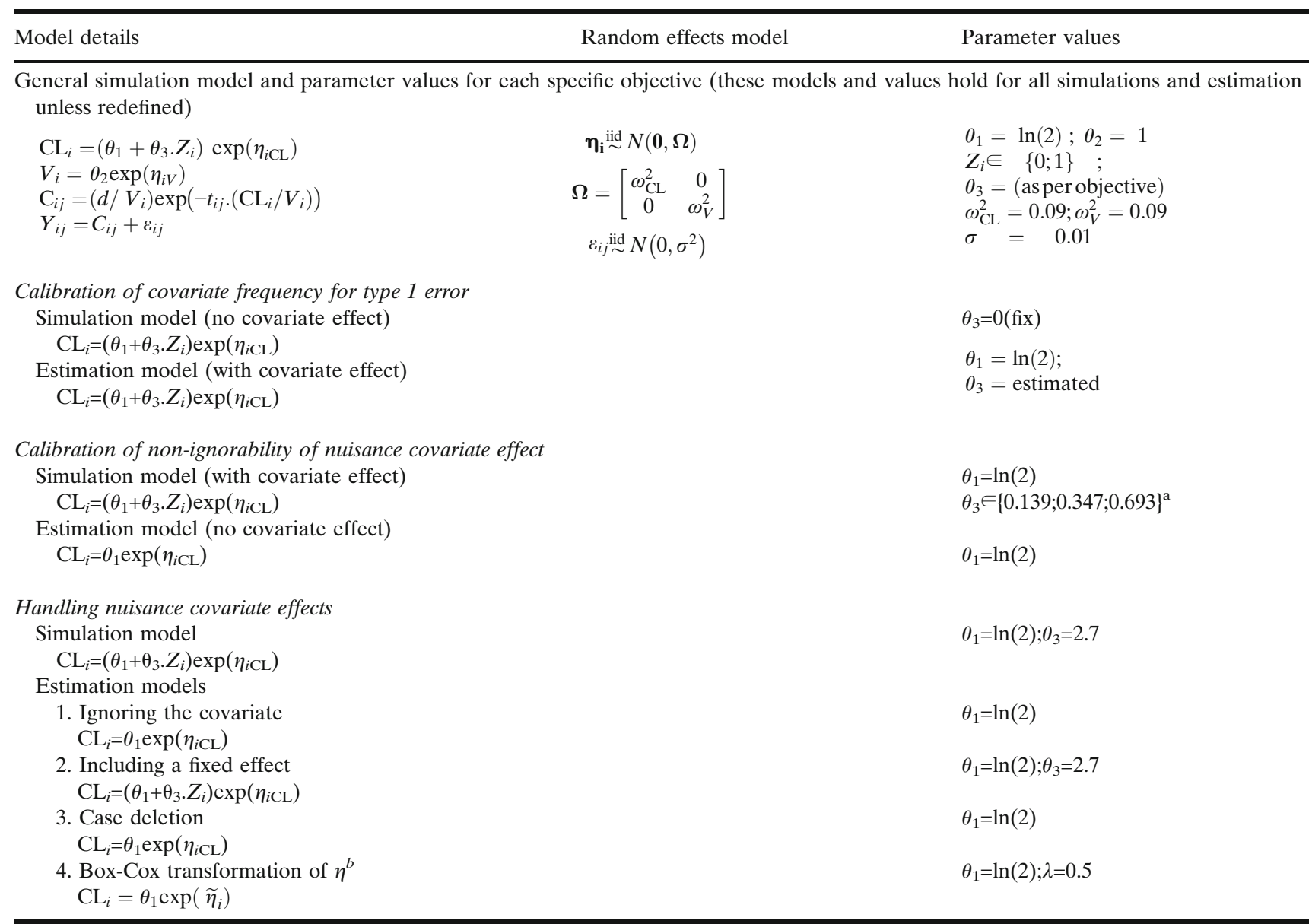

${ }^{a}$ Values for weak, medium and strong covariate effects, respectively

${ }^{b}$ As per Eqs. 3 and 4 in the text

\section{Handling Nuisance Covariate Effects}

To assess objective 3, an extreme example of a very strong covariate effect ( $+400 \%$ influence on CL) was considered similar to that found in Dansirikul et al. (7) with a frequency of $4 \%$, a frequency that could reasonably be expected in routine data sets. In this scenario, we considered an extreme case for simulation (albeit not uncommon in practice) based on the frequency and strength of the covariate to illustrate how nuisance covariates can be handled effectively. This setting provided a working example of a nuisance covariate. We included a total number of subjects of 100 for each simulation with a total of 100 replications. Table I shows the parameter values and models used for simulation and estimation of PK data with the title of the specific objective. The following four approaches were considered for handling the nuisance covariates:

Ignoring the covariate (IC) - in this approach, the covariate was not included in the data analysis and all patients were included in the dataset (i.e. that the patient had the covariate in question that was ignored in the analysis).

Addition of a nuisance fixed effect parameter (FE)-here, the covariate was included in the data analysis for all patients and a fixed effect parameter was added to account for the influence of the effect.
Box-Cox transformation of between subject random effects $(\eta)(\mathrm{BC})$ - in this approach, the distribution of betweensubject variability $(\eta)$ was assumed to arise from a transformed normal distribution that was defined to have heavier tails as per Eq. 4. The between-subject random effect $\eta_{i}$ is assumed to be normally distributed with mean zero and variance $\omega^{2}$. A Box-Cox transformation of $\eta$ involved calculation of a transformed $\widetilde{\eta}_{i}$ from a normally distributed $\eta_{i}$ and an estimated shape parameter $(\lambda)$ as per Eq. 4 (see 8). Absolute values of $\lambda$ less than 1 provide heavier tails, whereas $\lambda$ values greater than 1 provide constricted tails. This transformed $\widetilde{\eta}_{i}$ is then related to the parameter of interest as per Eq. 1 in place of $\eta_{i}$.

$\eta_{i} \sim N\left(0, \omega_{i}^{2}\right)$

$\widetilde{\eta}_{i}=\frac{\left(\left(e^{\eta_{i}}\right)^{\lambda}-1\right)}{\lambda}$

Case deletion (CD) - in this approach, the patients who had the covariate of interest were deleted from the data set prior to conducting the data analysis. 


\section{RESULTS}

\section{Calibration of Covariate Frequency that Is Associated with Type 1 Error Inflation}

The results for calibration are shown in Fig. 1, and it is observed that as the frequency of the covariate decreases, then there is an increase in type 1 error from the nominal value. When the frequency of the covariate was less than $20 \%$ with a total sample size of 100 , the type 1 error was above the nominal value of 0.05 . When the total sample size increased to 1000 , the frequency of the covariate showing type 1 error inflation was less than $10 \%$. However, with a sample size of 20 , the type 1 error was above the nominal value of 0.05 even with $50 \%$ frequency of the covariate.

\section{Calibration of Non-ignorability of a Covariate Effect}

The second specific objective calibrates the size of the effect at which the covariate becomes non-ignorable. This was studied using weak, moderate and strong covariate scenarios. For this specific objective, a total sample size of 100 was considered based on the type 1 error observed in the specific objective 1 . For the weak covariate effect, it was found that the percent mean error for both the estimated parameters CL and variance of CL were negligible when the frequency of the covariate was less than $10 \%$ (upper row in Fig. 2). At greater values of the covariate effect (moderate and strong), the frequency of the covariate occurrence at which percent mean error was evident decreased. The covariate resulted in apparent mean error for moderate or strong covariate effects at frequency values greater than $5-10 \%$.

\section{Handling Nuisance Covariate Effects}

An example of a strong covariate effect was considered with a covariate frequency of $4 \%$. Figure 3 shows the results in terms of percent mean error for CL and variance of CL for various approaches evaluated to accommodate the nonignorable nuisance covariate. It was seen that including the subject but ignoring the covariate resulted in prediction mean error for both the CL and variance of CL. Addition of a fixed effect parameter resolved the mean error, and accurate estimates were shown for both CL and variance of CL. The

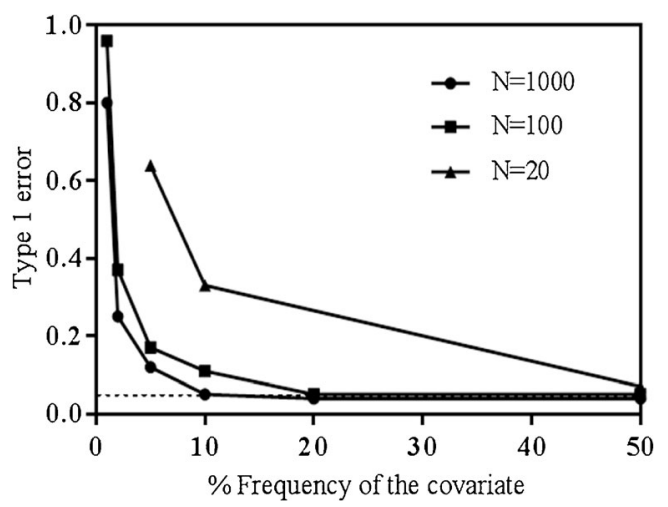

Fig. 1. Calibration of covariate frequency that is associated with type 1 error inflation with various sample sizes of 20,100 and 1000. The horizontal line indicates the nominal type 1 error value of 0.05 as the reference estimated covariate effect parameter was 2.94 (confidence interval 2.5 to 3.4 ), similar to the nominal value of 2.7. A BoxCox transformation of $\eta$ showed no prediction mean error for CL, but there was a slightly attenuated upward prediction of mean for the variance of CL. Case deletion showed no percent mean error for both these parameters. In the current case, $\mathrm{V}$ was found to be marginally less precisely estimated with case deletion with minimal effect on other parameters (Fig. 4). It is noted that the SE values were higher for CL and the between-subject variance on CL for both the method of ignoring the covariate but including the patient and the BoxCox transformation. The latter is likely to be due to the additional parameter for estimation. Interestingly, addition of a fixed effect parameter did not decrease precision of parameter estimation which suggests that the nonlinearity of $\lambda$ and the way it enters the statistical model for the random effects may come at greater cost in case of Box-Cox transformation.

\section{DISCUSSION}

Low-frequency covariates may often be observed in large data sets particularly in large late phase clinical trials and post-marketing evaluation studies. These data are then often explored to quantify exposure response relationships that can incorporate rare effects. This work and the work of Ribbing et al. (1) show that weak covariate effects are difficult to be determined accurately. The present study was intended to identify the circumstances in which a covariate is likely to be nuisance as well as evaluating various approaches of handling these covariates.

In this work, the influence of type 1 error was related to both the frequency of the covariate and the number of subjects in the overall population. The increase in type 1 error is more prominent at frequencies less than $5 \%$ which is likely to be problematic for rare genotypes or unexpected concurrently administered medicines leading to interactions. The work here was conducted in a nonlinear mixed effects modelling framework, and hence, exact solutions to standard statistical problems are generally not available. It is likely these findings will be dependent on the structural models chosen and the variance structures considered. However, it is seen that the works of several authors (3-5,911) show similar findings which are suggestive that these results may be generalizable, albeit with caution.

It is evident that consideration of the frequency of a rare covariate must be weighed against strength of its effect. Weak low-frequency covariate effects, defined here as an effect on the parameter of interest of $<20 \%$, appear to be ignorable, in that they do not cause significant parameter mean estimation error. However, strong and very strong covariate effects, $>100 \%$ increase in the parameter values or the corresponding relative decrease in the parameter value, appear nonignorable. As expected, the level of percent mean error in the parameter values depends on the frequency and strength of the effect.

Various approaches to handle nuisance covariates were evaluated in the setting of an extreme example of a very strong covariate effect. The various approaches evaluated were ignoring the covariate but including the patient in the dataset (IC), addition of a fixed effect parameter (FE), 
$C L$
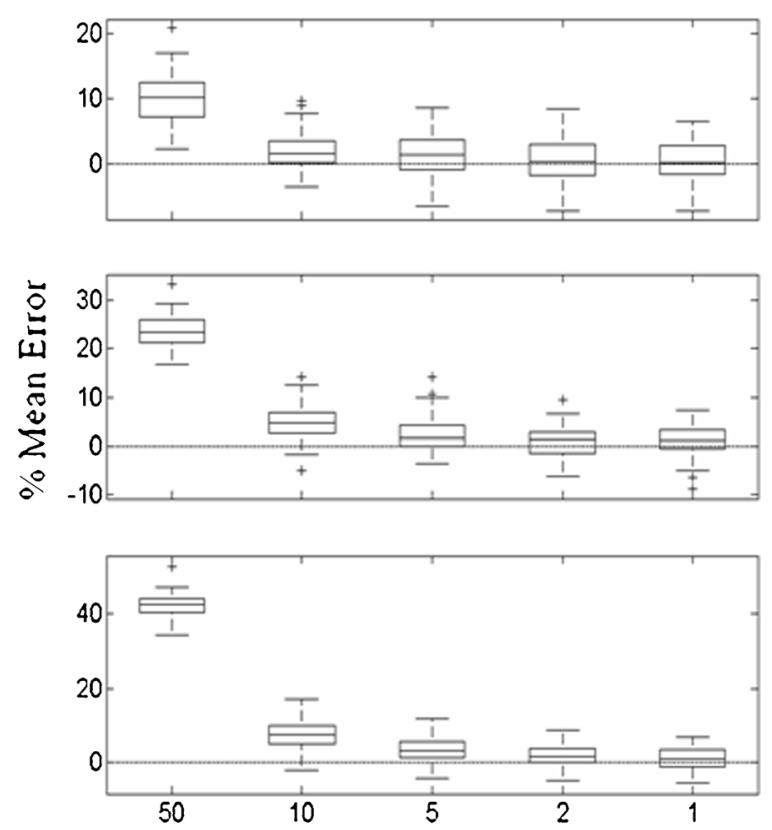

Variance of $\mathrm{CL}$
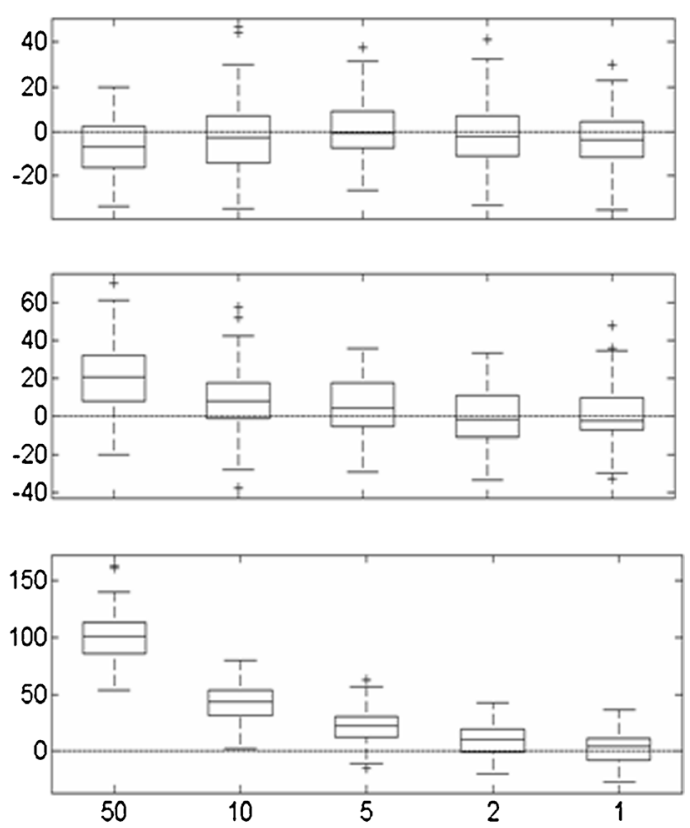

$\%$ Frequency of the covariate

Fig. 2. Calibration of non-ignorability of nuisance covariate effect assuming a weak covariate effect (+20\% influence on CL) with varying frequencies of the covariate (top panels), a moderate covariate effect $(+50 \%$ influence on $\mathrm{CL})$ with varying frequencies of the covariate (middle panels) and a strong covariate effect $(+100 \%$ influence on CL) with varying frequencies of the covariate (lower panels). The left plots show the percent mean error for CL and right plots the percent mean error for between-subject variance of CL. The horizontal line indicates the reference line for zero percent mean error. Sample size $=100$ for each scenario studied

accounting for heavy-tailed distribution through Box-Cox transformation of $\eta(\mathrm{BC})$ and finally case deletion, i.e. ignoring the covariate by deletion of patient data from dataset $(\mathrm{CD})$.
$\mathrm{CL}$

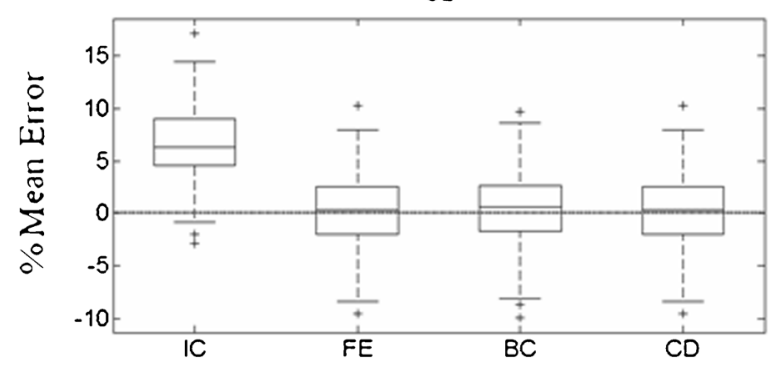

$\checkmark$

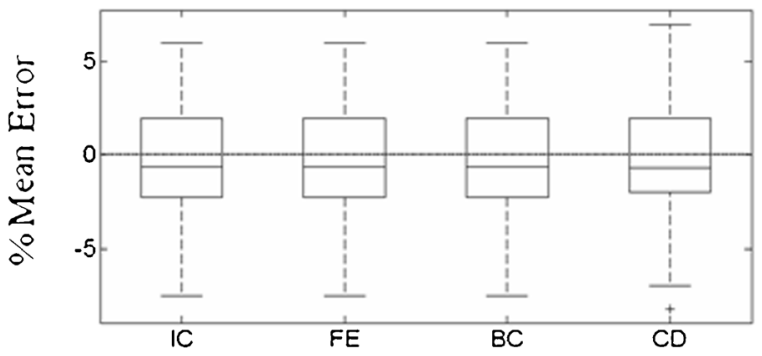

Variance of $\mathrm{CL}$

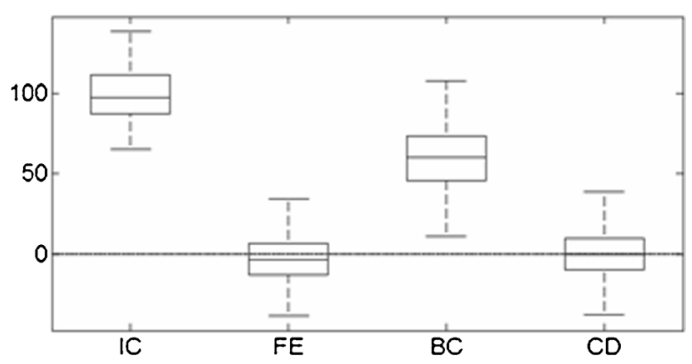

Variance of $V$

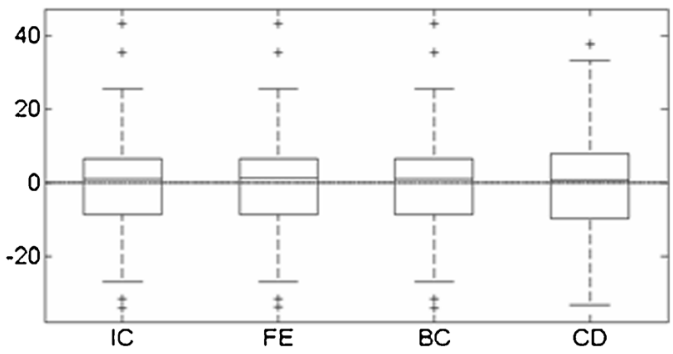

Fig. 3. Evaluation of methods to handle a non-ignorable nuisance covariate with the top panels showing percent mean error for CL and between-subject variance of $\mathrm{CL}$ and bottom panels showing percent mean error for $\mathrm{V}$ and between-subject variance of V. The horizontal line indicates the reference line for zero percent mean error. The abbreviations in the plot denote $I C$ ignore covariate but include patient, $F E$ include a fixed effect parameter, $B C$ use a heavy tailed Box-Cox transformation of $\eta, C D$ case deletion 

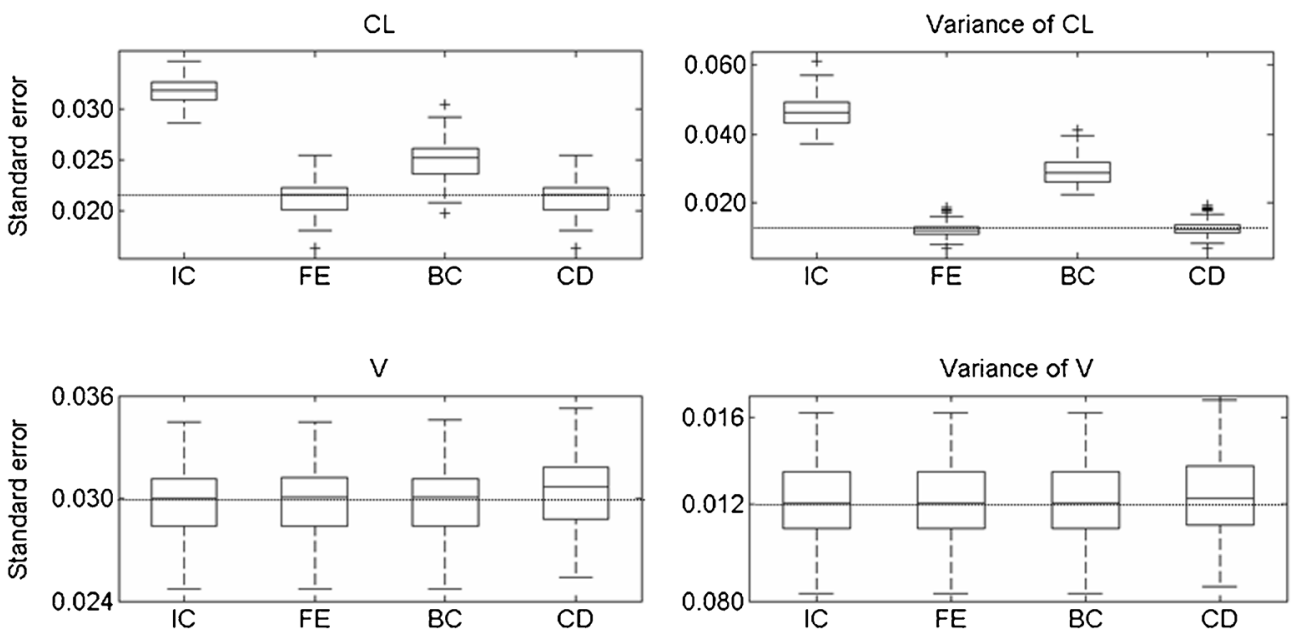

Fig. 4. Box plot of standard error estimates for CL (top left), between-subject variance of CL (top right), V (bottom left) and between-subject variance of $\mathrm{V}$ (bottom right). The abbreviations in the plot denote IC ignore covariate but include patient, $F E$ include a fixed effect parameter, $B C$ use a heavy tailed Box-Cox transformation of between-subject random effect $(\eta), C D$ case deletion. The horizontal lines indicate reference lines that help the standard errors for CD approach to compare with other approaches studied

It was observed that ignoring the covariate but including the patient in the dataset resulted in greater percent mean error for the parameter estimates relating to that covariate, i.e. in this work, the percent mean error related to CL and its associated variance of the random effect for CL. Naturally, this also has important implications for unobserved covariates which have strong effects. This latter case may occur in any analysis and result in a lack of interchangeability of the results of one analysis to another (see some discussion of this in Duffull 2013 et al. (12)). However in circumstances when the covariate is observed, it should be treated as non-ignorable. A pragmatic solution is to consider a fixed effect parameter/ model to account for the covariate. In our work, this provided a reasonable method for accommodating non-ignorability and eliminated percent mean error of the parameters. However, the resulting fixed effect parameter estimate itself is nuisance and should not be used for inferential purposes since its value may not be accurate and/or may itself be nonsignificant due to type 1 error. In the current study, the estimated mean value of the coefficient for the covariate was 2.94 compared to the nominal value of 2.7. A reliable identification of covariate is achieved after adjusting for the inflated type 1 error using a permutation test. An inflated value is not unexpected given the works of Ribbing et al. (1) and La Caze et al. (13) as is the case with nuisance covariates. The approach of Box-Cox transformation of $\eta$ was as effective at eliminating the percent mean error on the fixed effect parameter CL, but percent mean error remained for variance of CL. This latter finding is not however an appropriate conclusion since the variance of the random effect was subject to further transformation, and hence, the Gaussian distributional assumption on the original domain does not necessarily hold. We see in the work of Dansirikul et al. (7) that a multivariate log-t-distribution with low degrees of freedom reduced the between-subject variance of clearance in a Bayesian analysis. However, the work of Dansirikul et al. did not provide additional theoretical underpinning for handling low-frequency influential covariates. Case deletion showed no percent mean error for the parameters CL and variance of CL. However, a very slight increase in the imprecision of $\mathrm{V}$ was observed with this approach. This effect is minimal and is most likely to be due to the reduction in the number of subjects due to case deletion. Case deletion is useful if the focus is on the population value of CL, but this will have consequences in terms of precision of other parameter estimates (e.g. V). Case deletion, although a simple method for reducing percent mean error, is not desirable as it necessitates a change in the data set which poses logistical problems with data owners in formal clinical trial settings.

The approaches of case deletion and extra parameter addition have been investigated for missing categorical covariate data $(14,15)$. In their work, the categorical covariate is missing as opposed to the current work where the covariate is not missing. Hence, the current work can explore different approaches including fixed effects models that are not possible when the covariates are missing. It is also possible that the use of a mixture model will perform better than ignoring the covariate. Further, one can argue that a mixture model might be a good alternative to Box-Cox transformation for a discrete covariate. In this case, the FE method described here is equivalent to a mixture model but where the presence of the covariate is known. If the covariate is latent, then a mixture model where the probability of the presence of a covariate effect is estimated may perform well, but this is likely to depend on the frequency and strength of the covariate effect.

In the present evaluation, a Wald test was used for assessment of statistical significance. This provides a local assessment of significance associated with a parameter or set of parameter value(s). An alternative global measure of statistical significance could use a likelihood ratio test (LRT) or other similar statistic. The Wald test is less computationally intensive as the test can be performed on any given analysis without the need to repeat an analysis for the null model. This may have advantages for some covariate model building strategies. It is seen that type 1 error rates are less pronounced with the LRT method, when using NONMEM with the settings as FOCE with INTERACTION (3-5,9-11). 
This finding is also likely to be design dependent, and further work is required here to understand the influence of nuisance and non-ignorable covariates when model-based decisions are undertaken using the LRT. While the same general results are expected, caution should be used if extrapolating the specific covariate frequencies explored in this work to other circumstances where different statistical tests are used. Though we used an intensive sampling design for simulation and estimation in this work, we believe our results could be generalized to other settings. We would caution, however, that while the findings are likely to remain similar, the scale of the influence may vary based on models and designs. This work can also be extended to include categorical covariates. It is expected that simply accounting for the distribution of random effects is not enough for less frequent covariates commonly observed in pharmacogenetics. This work provides some general findings relating to nuisance covariates and non-ignorability that can be generalized to other model building settings in PKPD. Our findings regarding methods for handling these low-frequency methods while are likely to be generalizable should be investigated on a case by case basis in circumstances that involve more complicated covariate relationships. Further, we have not determined power in the current work and it will be low, as per observations from Ribbing (1), as both the sample size and frequency of the covariate will influence the power to detect a covariate.

\section{CONCLUSION}

In conclusion, nuisance covariate effects can be handled via addition of a fixed effect covariate model but the coefficient that pertains to the covariate effect should not be used for inference. The approach of allowing for a heavytailed distribution for inclusion of the covariate through a Box-Cox transformation of between-subject random effects was effective for reducing fixed effects parameter percent mean error, but the variance of the random effects is affected by the transformation. Case deletion and ignoring the covariate are not recommended.

\section{ACKNOWLEDGMENTS}

CL received University of Otago Post-graduate Scholarship and was awarded University of Otago Post-graduate publishing bursary during the preparation of this manuscript.

\section{REFERENCES}

1. Ribbing J, Jonsson EN. Power, selection bias and predictive performance of the Population Pharmacokinetic Covariate Model. J Pharmacokinet Pharmacodyn. 2004;31(2):109-34.

2. Bertrand J, Comets E, Chenel M, Mentré F. Some alternatives to asymptotic tests for the analysis of pharmacogenetic data using nonlinear mixed effects models. Biometrics. 2012;68(1):146-55.

3. Nakade S, Nishibori A, Okamoto H, Higuchi S. Statistical evaluation of clinical trial design for a population pharmacokinetic study-a case study. Drug Metab Pharmacokinet. 2004;19(5):381-9.

4. Kowalski KG, Hutmacher MM. Design evaluation for a population pharmacokinetic study using clinical trial simulations: a case study. Stat Med. 2001;20(1):75-91.

5. Lee PI. Design and power of a population pharmacokinetic study. Pharm Res. 2001;18(1):75-82.

6. Laouénan C, Guedj J, Mentré F. Clinical trial simulation to evaluate power to compare the antiviral effectiveness of two hepatitis $\mathrm{C}$ protease inhibitors using nonlinear mixed effect models: a viral kinetic approach. BMC Med Res Methodol. 2013;13(1):60.

7. Dansirikul C, Morris RG, Tett SE, Duffull SB. A Bayesian approach for population pharmacokinetic modelling of sirolimus. Br J Clin Pharmacol. 2006;62(4):420-34.

8. Petersson KJ, Hanze E, Savic RM, Karlsson MO. Semiparametric distributions with estimated shape parameters. Pharm Res. 2009;26(9):2174-85.

9. Bertrand J, Comets E, Laffont CM, Chenel M, Mentré F. Pharmacogenetics and population pharmacokinetics: impact of the design on three tests using the SAEM algorithm. J Pharmacokinet Pharmacodyn. 2009;36(4):317-39.

10. Gobburu JV, Lawrence J. Application of resampling techniques to estimate exact significance levels for covariate selection during nonlinear mixed effects model building: some inferences. Pharm Res. 2002;19(1):92-8.

11. White DB, Walawander CA, Liu DY, Grasela TH. Evaluation of hypothesis testing for comparing two populations using NONMEM analysis. J Pharmacokinet Biopharm. 1992;20(3):295-313.

12. Duffull SB, Wright DF. What do we learn from repeated population analyses? Br J Clin Pharmacol. 2015;79(1):40-7.

13. La Caze A, Duffull S. Estimating risk from underpowered, but statistically significant, studies: was APPROVe on TARGET? J Clin Pharm Ther. 2011;36(6):637-41.

14. Keizer RJ, Zandvliet AS, Beijnen JH, Schellens JH, Huitema AD. Performance of methods for handling missing categorical covariate data in population pharmacokinetic analyses. AAPS J. 2012;14(3):601-11.

15. Kaila N, Straka RJ, Brundage RC. Mixture models and subpopulation classification: a pharmacokinetic simulation study and application to metoprolol CYP2D6 phenotype. J Pharmacokinet Pharmacodyn. 2007;34(2):141-56. 